(C) 2014 IEEE. Personal use of this material is permitted. Permission from IEEE must be obtained for all other uses, in any current or future media, including reprinting/republishing this material for advertising or promotional purposes, creating new collective works, for resale or redistribution to servers or lists, or reuse of any copyrighted component of this work in other works. 


\section{Effects of Quality of Service Schemes on the capacity and dimensioning of LTE Networks}

\author{
Fatima Furqan \\ INEXT Centre for Innovation in IT \\ Services and Applications \\ University of Technology, Sydney \\ Fatima.furqan@student.uts.edu.au
}

\author{
Doan B. Hoang \\ INEXT Centre for Innovation in IT \\ Services and Applications \\ University of Technology, Sydney \\ Doan.Hoang@uts.edu.au
}

\author{
Iain B. Collings \\ ICT Centre, CSIRO, Australia. \\ iain.collings@csiro.au
}

\begin{abstract}
With high data rate and mobility support wireless networks are becoming an integral part of the ubiquitous broadband access. Appropriate dimensioning of the wireless access networks is essential to satisfy users' Quality of Service (QoS) requirements. Once the network is dimensioned, dynamic variations in traffic or population distribution can affect the capability of the network to deliver the agreed QoS of connections. Consequently, re-dimension the network may be necessary. Current researches do not discuss the effects of QoS schemes on the capacity of the network. In this paper, we investigate the impact of QoS schemes on the capacity of the network. The objective is to determine the capability of the network to deal with the variations in the demography of the covered area and the user's traffic profile with the proposed QoS schemes including Congestion Control (CC) and Radio Admission Control (RAC). Different scenarios are presented to evaluate the effects of QoS schemes on the capacity of the network. This investigation will assist network operators to determine the point after which the network needs to be redimensioned.
\end{abstract}

\section{Keywords- LTE; Coverage; Capacity; overheads; Dimensioing}

\section{INTRODUCTION}

For high speed infrastructure that covers the whole continent a combination of wired and wireless technologies is necessary due to cost and physical limitations. For example, the Australian National Broadband Network (NBN) is taking place to connect all Australian premises. The infrastructure consists of Fiber To The Premises (FTTP) and Broadband Wireless Access (BWA) to cover 93\% and 4\% of the premises, respectively. The rest of the country is covered by satellite broadband access. Long Term Evolution (LTE) technology is considered for BWA as it evolves towards $4^{\text {th }}$ Generation (4G), which can deliver 100Mbps and beyond.

This paper focuses on LTE technology. To guarantee the service differentiation and provision of Quality of Service (QoS), LTE employs the concept of bearer. A bearer identifies packet flows from one network element to another. The QoS profile of each bearer includes QoS Class Identifier (QCI) and Allocation and Retention priority (ARP). QCI defines the forwarding treatment of IP packets received on a bearer. In situations when resources are limited, admission control uses ARP to determine whether a new bearer can be established. LTE supports two types of bearers, Guaranteed Bit Rate (GBR) and Non Guaranteed Bit Rate (non GBR). The Bandwidth (BW) allocation to bearers is managed with two parameters, Guaranteed Bit Rate (GBR) and Maximum Bit Rate (MBR).

LTE physical supports transmission in both Time Division Duplex (TDD) and Frequency Division Duplex (FDD) modes. Most of the current implementations of LTE are in FDD mode, so we confine our discussion to FDD. In FDD mode, Frame structure type 1 is used. As indicated in Fig. 1, each radio frame is $10 \mathrm{~ms}$ long and consists of 10 subframes of length $1 \mathrm{~ms}$ each. Each subframe consists of 2 slots of length $0.5 \mathrm{~ms}$ each. Each slot consists of either 6 or 7 OFDM symbols for extended or normal cyclic prefix, respectively [1].

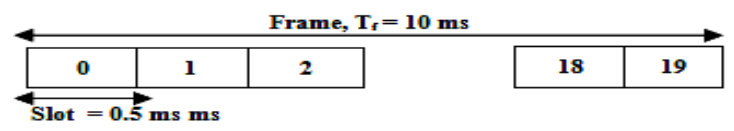

Fig. 1. Frame Structure Type 1, Reproduced from [1]

In frequency domain, each slot represents a Resource Block (RB) and consists of 12 subcarriers each $15 \mathrm{kHz}$ wide. A pair of two RBs (or a Transport Block (TB)) is the minimum unit used by the scheduler for allocations. A single subcarrier for one symbol duration is referred to as a Resource Element (RE).

The wireless broadband portion of the NBN covers many communities of different shapes and sizes from small areas with high population density to vast geographical areas, which are sparsely populated. Clearly, appropriate dimensioning of the whole access network is crucial to ensure the overall cost is minimized under the constraint that the specified data rates are guaranteed to all users regardless of their geographical locations. When performing network dimensioning, the bandwidth requirements of network connections are planned based on the condition that the network traffic is predetermined with limited room for variations. Whereas, some services like file transfer and email which employs Transmission Control Protocol (TCP) at the transport layer are elastic. They adjust their source rates according to the available bandwidth in the network [2]. In [3, 4], we proposed Fair Intelligent Congestion Control (LTE-FICC) and Fair Intelligent Admission Control (LTE-FIAC). These QoS schemes utilize the elastic feature of the TCP traffic and the flexibility in bandwidth allocation between the MBR and GBR of a bearer in the latest releases of 3GPP. These schemes employ bandwidth adaptation (BA) algorithms to accommodate the variations in traffic demand and load at the 
core network. Consequently, for a given network configuration, they impact the ability of the network to deal with the dynamic variations in the network.

Several results on dimensioning of LTE networks have been made available in other publications. [5] presents a coverage and capacity analysis for LTE networks for the city of Dhaka. [6] explains different steps of dimensioning procedure. It presents models and methods for the capacity and coverage planning of LTE networks. [7] explains dimensioning models for LTE access transport network including X1 and S1 interfaces. The authors consider both elastic and real time traffic in analysis. [8] discusses the effect of scheduling, antenna diversity and Multiple Input and Multiple Output (MIMO) on the capacity of VoIP in LTE. All of these schemes confine their discussions to only dimensioning process. They do not deal with the changes in network characteristics once the network has been dimensioned. For example, the load at the core network (EPC), which connects base stations to internet (as shown in Fig. 2) increases or the traffic demand of a cell increases beyond the forecast due to changes in population density or application distributions. In these situations, a network without appropriate QoS schemes may not be able to manage these dynamic changes and as a result QoS of connections degrades. Consequently, it imposes a requirement on network operators to re-dimension the network. Whereas, with employment of appropriate QoS schemes the network operators are able to manage the increase in data demand and load at the core network to a certain extent, without the need to re-dimension.

This paper focuses on several aspects, which have not received much attention for LTE capacity estimation. First, we explain LTE control channels and protocol overheads (OHs), which significantly affect the capacity of the network. Second, as scheduling has enormous affect on the amount of protocol overhead. In current analysis, the protocol overhead per second for each application is determined. Third, the paper discusses the effect of bandwidth and applications distribution on the resource utilization and the capacity of the network. Fourth and foremost, the paper discusses the capacity of a cell in terms of users supported with our proposed QoS schemes. It facilitates to determine the point to which the network can cope with the dynamic fluctuations in application demand, population distributions; and the load at the core network, without the need to re-dimension while also maintaining the QoS of connections. In doing so the paper suggests a novel and efficient way to the network operators to estimate the number of users which can be supported under dynamically varying network load and demand parameters, by employing specific QoS schemes at the base station.

The paper is organized as follow: The LTE system model and traffic analysis are discussed in section II. Coverage of LTE networks is discussed in section III. Section III gives a description of the system and protocol overheads. It also discusses the effect of bandwidth, application distribution (AD) and most the proposed QoS schemes on capacity of the network. Finally section IV is devoted to conclusion.

\section{SYSTEM AND TRAFFIC MODELS}

\section{A. System Model of LTE}

The NBN employs fixed wireless to cover the areas where fiber roll out is not technically or financially feasible.

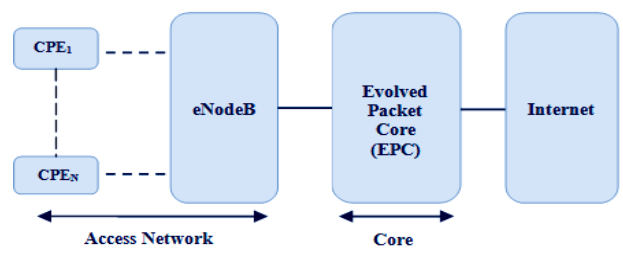

Fig. 2. LTE Network Architecture

1) Customer Premises Equipment (CPE):

$\mathrm{CPE}$ is installed at the user's premises. The NBN Co CPE consists of an indoor and an outdoor unit. The outdoor fixed antenna is installed at the rooftop of the customer's premises to provide Line of Sight (LoS) connectivity with the antenna of base station, called enhance NodeB (eNodeB) in LTE [9].

2) LTE Base Station (eNodeB)

A fixed wireless facility with three antennas is installed. Each antenna covers a certain area to maximize the signal strength.

3) Spectrum

NBN Co has selected $2.3 \mathrm{GHz}$ and $3.4 \mathrm{GHz}$ spectrum bands for deployment of Time Division LTE (TD-LTE).

\section{B. Traffic Profiles}

Among the key inputs to capacity planning of a cell are the type and distribution of applications in use. Services in consideration include Voice-over-Internet Protocol (VoIP), video streaming, multiplayer gaming, file transfer and web. This analysis is general and can be applied to other types and distributions of applications.

- VoIP: It is used for voice calls over packet switched networks. In current analysis, VoIP traffic is generated using G.711 encoder scheme with a voice payload size of 160 bytes and an interval of $20 \mathrm{~ms}$.

- Streaming: It is used for video conferencing and videos upload/download. Its data rate depends on the quality and size of the display. We assume a frame size of 128 X 120 pixels. The frame inter-arrival time is set to $160 \mathrm{~ms}$.

- Online Gaming: It allows players to play games online over the internet. In current analysis, data rate of online gaming is set to $50 \mathrm{kbps}$.

- File Transfer: FTP session is used to download or upload files. The file size is set at $1 \mathrm{Mbps}$ and the inter-request time is 4.0 second.

- Web: It specifies the HTTP sessions between the client and server. In current analysis, the web browser uses HTTP 1.1 protocol. The web page properties and the main attributes of the traffic are given in table I.

TABLE I. PARAMETERS OF WEB TRAFFIC

\begin{tabular}{|l|l|}
\hline Parameters & Values \\
\hline Main Page Size (Bytes) & 10710 \\
\hline Embedded Object Size (Bytes) & Uniform (4000-8000) \\
\hline Number of Embedded objects & 10 \\
\hline Reading Time (secs) & 10 \\
\hline Request size (Bytes) & 350 \\
\hline
\end{tabular}


To appropriately define the applications demand distribution, users in a cell are grouped as follows.

- Households: The household users normally use VoIP, streaming to upload/download videos or for IPTV, gaming and web services.

- Public and Health Services: The public and health services offices usually employ the web and VoIP services.

- Business: The businesses at any level mostly utilize the applications of web, VoIP, streaming for video conferencing and file transfer.

Keeping in view the demand of each user-group, two application distributions in table II are applied for capacity analysis. AD-1 covers the areas, which have relatively more businesses, health and public service offices. Whereas, AD-2 covers the areas that have more households, which relatively utilize more streaming services to upload/download videos.

TABLE II.

Application Distribution

\begin{tabular}{|l|l|l|l|l|l|}
\hline Application & QCI & $\begin{array}{l}\text { MBR } \\
(\mathbf{K b p s})\end{array}$ & $\begin{array}{l}\text { GBR } \\
(\mathbf{K b p s})\end{array}$ & $\left.\mathbf{( A}_{\mathbf{D}}-\mathbf{1}\right)$ & $\left.\mathbf{( A}_{\mathbf{D}}-\mathbf{2}\right)$ \\
\hline Streaming & 2 & 850 & 850 & $20 \%$ & $40 \%$ \\
\hline VoIP & 1 & 64 & 64 & $30 \%$ & $10 \%$ \\
\hline Online Gaming & 3 & 50 & 50 & $20 \%$ & $20 \%$ \\
\hline Web & 4 & 32 & 15 & $20 \%$ & $20 \%$ \\
\hline FTP & 4 & 256 & 50 & $10 \%$ & $10 \%$ \\
\hline
\end{tabular}

\section{NETWORK DIMENSIONING}

The network dimensioning activities include coverage analysis and capacity estimation. These tasks allow network operators to determine the overall site configurations in terms of total number of base stations and capacity of the core network required to guarantee data rate demands of the area of interest.

\section{A. Coverage Analysis}

This section covers the estimation of cell radius of LTE networks. For the coverage estimation, we have adopted most of the parameters suggested by NBN for the deployment of BWA [9] in Australia. As discussed in [10], coverage (CVG) is function of a number of parameters: transmit power $\left(\mathrm{P}_{\mathrm{Tx}_{\mathrm{x}}}\right)$, transmitter antenna gain $\left(\mathrm{G}_{\mathrm{Tx}}\right)$, receiver antenna gain $\left(\mathrm{G}_{\mathrm{Rx}}\right)$, Signal-to-Noise Ratio (SNR), frequency (f), eNodeB's antenna height $\left(\mathrm{h}_{\mathrm{b}}\right), \mathrm{CPE}$ antenna height $\left(\mathrm{h}_{\mathrm{CPE}}\right)$ and the bandwidth $(\Delta \mathrm{f})$ as shown in (1). This section discusses the effect of the frequency and bandwidth on the coverage using the empirical path loss model.

$$
\mathrm{CVG}=f\left(P_{T x}, G_{T x}, G_{R x}, S N R, f, h_{b}, h_{C P E}, \Delta \mathrm{f}\right)
$$

To calculate a cell radius, COST-231 Hata model [4] is used for the frequencies of $2.3 \mathrm{GHz}$ and $3.4 \mathrm{GHz}$. By applying an eNodeB antenna height of $40 \mathrm{~m}$ and $\mathrm{CPE}$ antenna height of $6 \mathrm{~m}$, the closed form to determine the path loss for urban, suburban and rural areas using Cost-231 Hata model are given in (2), (3) and (4), respectively.

$$
\begin{aligned}
& P L_{\text {Cost231-Hata }}=127.7+34.78 \log _{10}(d) \\
& P L_{\text {Cost231-Hata }}=1 \text { sub }
\end{aligned}
$$

$$
P L_{\text {Cost231-Hata }} \text { rural }=103.5+34.78 \log _{10}(d)
$$

Where ' $d$ ' is the distance between an eNodeB and a CPE. PL refers to path loss and is determined using (5).

$$
P L=\left(P_{T x}+G_{T x}+G_{R x}-L_{\text {others }}-R_{S S}\right)
$$

The $\mathrm{P}_{\mathrm{Tx}}$ of an eNodeB for the Downlink (DL) is set at 46 $\mathrm{dBm}$. For the Uplink (UL), the transmit power of a CPE is set at $23 \mathrm{dBm}$. The eNodeB deploys 3 sector antennas, so its antenna gain is set at $15 \mathrm{dBi}$ including the feeder loss. The antenna gain of a CPE is defined as $10 \mathrm{dBi}$ as it's a fixed wireless terminal and uses an outdoor directed antenna [11]. $\mathrm{L}_{\text {others }}$ refers to other losses in the network and includes Inference Margin (IM), body and indoor losses. UL is orthogonal, so there is no intra cell interference. The NBN frequency plan is to re-use three frequencies across the network. Hence, each of the three sectors of an eNodeB deploys a unique channel and there is no co-channel interference in a cell [9]. Consequently, the IM is set to $0 \mathrm{~dB}$. The antenna is fixed outdoor so the body and indoor losses are also set to $0 \mathrm{~dB}$. In $(5)$, Receiver Sensitivity $\left(\mathrm{R}_{\mathrm{ss}}\right)$ refers to the minimum received power required at the receiver to guarantee a given Block Error Rate (BLER). For OFDMA PHY, $\mathrm{R}_{\mathrm{ss}}$ is determined using (6).

$$
R_{S S}=-174+10 \log _{10}(\Delta f)+N F+S N R_{R x}
$$

SNR in (6) determines Channel Quality Indication (CQI) and in turn the Modulation and Coding Scheme (MCS) that is to be used for the transmission. MIMO antenna diversity reduces the effect of multipath fading on the SNR of the received signal [12]. For the coverage analysis, the DL transmit diversity is set at $2 \times 2$ and UL transmit diversity is set at $1 \times 2$. So, the paper employs the SNR values determined in [12] for the transmit diversity of $2 \times 2$ corresponding to $10 \%$ BLER. In (6), NF refers to Noise Figure at the receiver. It is set at 9 and $5 \mathrm{~dB}$ for the DL and UL, respectively. $\Delta$ f refers to system bandwidth in Hz. LTE supports scalable bandwidth from $1.4 \mathrm{MHz}$ to $20 \mathrm{MHz}$.

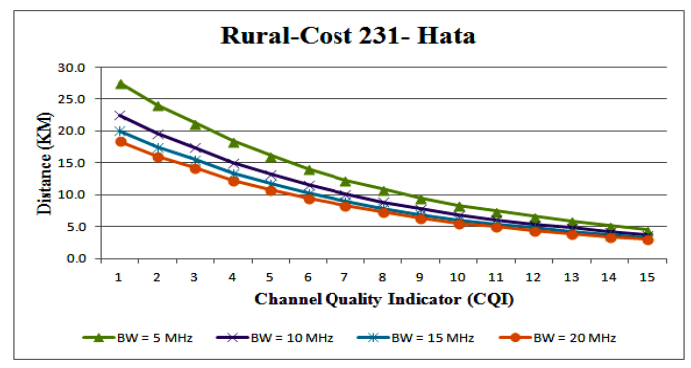

Fig. 3. Cell Radius for various Channel Quality Indicators

Fig. 3 shows cell radius of rural area for different values of CQIs. It gives cell radius for $2.3 \mathrm{GHz}$ frequency with 5, 10, 15 and $20 \mathrm{MHz}$ bandwidths, respectively. It shows the cell radius reduces for higher values of CQIs. It is because SNR is higher only close to an eNodeB. The estimations demonstrate that a cell radius of suburban and urban is $52 \%$ and $80 \%$ smaller compared to a cell radius of rural area given in Fig.3. Estimations also indicate that for $3.4 \mathrm{GHz}$ frequency cell 
radius is $32 \%$ smaller compared to a cell radius of $2.3 \mathrm{GHz}$. So, lower the frequency, the higher is the cell radius.

Fig. 3 also indicates that for any specific CQI when the bandwidth is doubled, a cell radius reduces by around 0.2 percent. Hence, lower bandwidths gives better cell coverage compared to higher bandwidths due to the receiver sensitivity. The $10 \cdot \log 10(\Delta \mathrm{f})$ component of the receiver sensitivity (6) encompasses all subcarriers in the bandwidth. Consequently, when the bandwidth increases, the minimum power required at the receiver $\left(\mathrm{R}_{\mathrm{ss}}\right)$ also increases and results in reduced cell radius.

Normally, the site configurations derived from the coverage estimation remains the same until or unless the coverage area converges from rural to suburban or suburban to urban. So, our analysis is restricted to the factors that affect the supported capacity of the network.

\section{B. Capacity planning}

To estimate the number of users that can be supported with given traffic profiles, effective capacity of the network needs to be determined. The effective capacity is the amount of resources in the network actually utilized for transmission of user's data. The effective capacity (CPC) of a cell is a function of frequency (f), bandwidth, frame duration $\left(\mathrm{T}_{\mathrm{f}}\right)$, SNR, modulation (E), code rate (CR), cyclic prefix (CP), and overhead $(\mathrm{OH})$ as shown in (7) [10]. The lower the value of $\mathrm{CP}$ and code rate, the higher is the capacity, as less redundant data is sent. Similarly, the usage of high capacity modulation gives high capacity as shown in [6].

$$
\mathrm{CPC}=f\left(\mathrm{f}, \mathrm{BW}, \mathrm{T}_{\mathrm{f}}, \mathrm{SNR}, \mathrm{E}, \mathrm{CR}, \mathrm{CP}, \mathrm{OH}\right)
$$

This paper considers the effects of control channels and protocol overheads on the effective capacity of the network. The paper also investigates the effect of bandwidth, application distribution, and QoS schemes employed at an eNodeB on the capacity of the network in terms of users supported with at least minimum guaranteed QoS.

\section{1) Control Channels Overhead}

Control channels carry control information in the cell. They have a direct impact on the resources available to the RAC to allocate to the users. Table III shows the number of TBs per second (TBPS) and REs per TB for FDD physical profile with 5, 10, 15 and $20 \mathrm{MHz}$ bandwidths, respectively. It clearly indicates that the number of TBPS increases as the bandwidth increases.

The number of TBPS for the UL is estimated taking into account TBs required by the Physical Uplink Control Channel (PUCCH) and Physical Random Access Channel (PRACH). PUCCH carries control information, such as user's Scheduling Request (SR), Hybrid ARQ (HARQ) ACK/NACK and CQI. PRACH carries user's Random Access (RA) preamble and offers an interface between unsynchronized CPEs and the uplink radio access [13]. We applied RA preamble format 0 . The number of RA resources per frame is set to 1 . In the current set up, 20 and 6 TBs per frame are set for PUCCH and PRACH respectively $[14,15]$.
Table III also shows the average number of REs per TB. On average there are $168(12 \times 7 \times 2)$ and 144 (12 X 6 X 2) REs per TB for normal and extended $\mathrm{CP}$, respectively. The estimation of REs takes into account the overhead of DL and UL reference symbols (pilot symbols). They are inserted in a subframe for the channel estimation and the signal demodulation at the receiver [13]. The reference symbols occupy 12 and 4 REs in each RB for UL and DL, respectively.

For DL, the estimation of REs per TB further takes into account the Physical Downlink Control Channel (PDCCH) symbols per subframe. PDCCH mainly carries DL assignments and UL grants, PRACH responses and UL power control commands. As per $3 \mathrm{GPP}$ specifications it can take 1,2 or 3 symbols per subframe. For the estimation of REs per TB, the PDCCH symbols per subframe are set at $3(21 \% \mathrm{OH})$. As according to the LTE specifications the TB to bits mapping table is constructed by considering 120 reference REs in the $\mathrm{TB}$, so an average number of REs per TB is estimated to match the specifications [15].

TABLE III. TRANSPORT BLOCKS PER SECOND AND RES PER TB

\begin{tabular}{|c|c|c|c|c|}
\hline & \multicolumn{4}{|c|}{ Bandwidth } \\
\hline BW & \multicolumn{2}{|c|}{5} & 10 \\
\hline TBPS & DL & UL & DL & UL \\
\hline Avg. RE per TB & 25000 & 22400 & 50000 & 47400 \\
\hline BW & 123 & 139 & 124 & 139 \\
\hline TBPS & DL & UL & DL & UL \\
\hline Avg. RE per TB & 75000 & 72400 & 100000 & 97400 \\
\hline \multicolumn{2}{|c|}{125} & 139 & 125 & 139 \\
\hline
\end{tabular}

The actual number of TBPS available in the admission procedure also depends on UL and DL loading factor. The loading factor indicates the fraction of the total resources available to the RAC. In this work, UL and DL loading factors are set at 1 . It indicates the maximum possible resources are available to the admission control algorithm for both UL and DL.

\section{2) Protocol Overhead}

A significant portion of the total resources, available to the RAC, is used to accommodate the protocol $\mathrm{OH}$. The total amount of protocol OH is based on the scheduler in use [16].

The Real-Time Transport Protocol/User Datagram Protocol/ IP (RTP/UDP/IP) and TCP/IP overheads (OH) per packet in an uncompressed mode are 40 and 60 bytes each for IPv4 and IPv6, respectively. The Packet Data Convergence Protocol (PDCP) layer at the top of the radio interface protocol stack compresses higher layers overhead. It uses Robust Header Compression (ROHC) protocol, which is specified by Internet Engineering Task Force (IETF). With ROHC the maximum compressed header sizes per packet are considered to be 4,2 and 4 bytes for RTP/UDP/IP, UDP/IP and TCP/IP, respectively [15]. The PDCP layer has a fixed overhead of 16 bits. The Radio Link Control (RLC) layer has overhead of 2 bytes. With segmentation or concatenation the RLC overhead is assumed to increase to 4 bytes. The Medium Access Control (MAC) layer overhead is 6 bytes. 
Also, the physical layer adds 3 bytes Cyclic Redundancy Check (CRC) checksum to data packet [17].

As discussed, protocol $\mathrm{OH}$ per second of each application heavily depends on the scheduler. The scheduler we considered in our analysis, serves the connections based on the delay requirements [15]. Instead of scheduling each service flow in every subframe, the scheduler holds its data for certain number of subframes hereafter referred to as "Interval". The Interval is determined based on the following equation.

$$
\text { Interval }=\frac{\text { delay_budget }}{\text { subframe_dur }}
$$

In (8), Subframe_dur is the duration of subframe. The delay_budget is an acceptable delay of the QoS class to which the service flow belongs. The services are mapped to QoS classes according to the mapping shown in table II.

Based on the Interval estimated in (8), Table IV provides the required data rate of each application to meet its data and protocol overhead requirements for $20 \mathrm{MHz}$ bandwidth.

TABLE IV. Protocol OHs with SCHEdUlER - $20 \mathrm{MHz}$

\begin{tabular}{|l|l|l|l|l|l|}
\hline & Streaming & VoIP & $\begin{array}{l}\text { Online } \\
\text { Gaming }\end{array}$ & FTP & Web \\
\hline $\begin{array}{l}\text { User-Data at IP } \\
\text { layer (Bytes) }\end{array}$ & 1377 & 640 & 250 & 1377 & 960 \\
\hline $\begin{array}{l}\text { Data+ Headers } \\
\text { +CRC (Bytes) }\end{array}$ & 1394 & 659 & 269 & 1394 & 979 \\
\hline Served_sub & 77 & 13 & 25 & 23 & 4 \\
\hline Total OH (kbps) & 10.0 & 1.9 & 3.8 & 3.5 & 0.41 \\
\hline Total rate (kbps) & 860.5 & 65.9 & 53.8 & 259.1 & 32.6 \\
\hline
\end{tabular}

For example, the streaming application has data requirements of $850 \mathrm{kbps}$. It's QCI is 2 and hence the delay budget is $150 \mathrm{~ms}$. The scheduler estimates to serve the service flow in every $120^{\text {th }}$ subframe, based on $80 \%$ of the interval estimated in (8) [15]. We took $80 \%$ of the interval in (8) to allow a margin for the delay at the core network. In current scenario, streaming generates a frame every $30 \mathrm{~ms}$. Hence, scheduler can send four video subframes in one subframe based on the delay requirements. This allows the scheduler to reduce the protocol overhead. Whereas, the maximum bits that can be served in a subframe depend on the MCS of CPE and bandwidth of the system. For example, $20 \mathrm{MHz}$ bandwidth and 64 QAM-3/5 MCS allows the maximum transmission of 3542 bytes per subframe [18]. Moreover, in LTE networks, GPRS Tunneling Protocol (GTP) tunnels carry bearers in an all IP based core network. The Maximum Transmission Unit (MTU) of an IP core is 1500 bytes. The GTP/UDP/IP and IPsec tunneling also add overheads to packet. To enable the packet to fit to an Ethernet interface Service Data Unit (SDU) of the core network, one way is to set the MTU at an IP layer of a CPE to be less than or equal to 1394 bytes for IPV4 (1358 bytes in case of IPv6 transport) [19]. As discussed, packet scheduled also accommodates protocol headers of maximum 20 bytes using ROHC and allows the transmission of approximately 1374 bytes of user's data per subframe.
Consequently, this requires the transmission of streaming application's data at every $14^{\text {th }}$ subframe to meet its throughput and delay requirements. So, the data flow needs to be served in 77 subframes hereafter referred as served sub. As a result, the protocol overhead is estimated to be $10.5 \mathrm{kbps}$ and the total rate requirement of the data flow increases to 860.5 kbps. The total required data rate for other applications is estimated in a similar way. The VoIP, online gaming and web applications generates relatively small packet sizes. The scheduler based on the delay requirements of each application concatenates its packets to reduce the protocol overhead.

Table IV clearly indicates that scheduler requires resources in addition to the data rates given in table II to cover the protocol overhead of connections. While, in our analysis we set the UL and DL loading factor to 1, which means all resources are available to RAC. Consequently, it keeps on allocating the left over resources to incoming connections without the consideration of protocol $\mathrm{OH}$. As a result, the QoS of connections degrades. So to ensure the QoS of all users, the GBR and MBR given in the table II should be updated to cover the amount of total protocol $\mathrm{OH}$ estimated in table IV.

To incorporate the fact that in reality varying channel conditions impact the capability to use a particular MCS, average cell throughput is defined using the following equation [6].

$$
A v g_{-} c e l l \_T H=\sum_{i=0}^{15} P_{S N R_{i}} * T H_{S N R_{i}}
$$

$\mathrm{P}_{\mathrm{SNR}_{\mathrm{i}}}$ is the probability of occurrence of an SNR value at a cell edge corresponding the CQI ' $\mathrm{i}$ '. $T H_{S N R_{i}}$ is the throughput with a specific MCS corresponding the CQI ' $i$ '. It also depends on the bandwidth of the system. To perform capacity analysis, we assumed a fixed network and uniform probability distribution for different MCS at $2.3 \mathrm{GHz}$ frequency.

\section{3) Capacity Estimation}

Capacity estimation determines total number of users supported for specific traffic types, distributions and system settings. For capacity estimation, frame duration is set to 10 $\mathrm{ms}$ and normal CP is employed. To analyze the impact of BW, application distribution and QoS schemes two application distributions $\mathrm{AD}-1$ and $\mathrm{AD}-2$ given in table II are employed. For simplicity of analysis, capacity estimation is performed for an eNodeB with a $1 \times 1$ antenna with $20 \mathrm{MHz}$ channel. Also, it does not consider the affect of H-ARQ.

TABLE V. NumBer of SUPPORTED USERS AND APPLICATIONS DATAUSAGE FOR AD-1

\begin{tabular}{|r|r|r|r|r|r|r|}
\hline & \multicolumn{1}{|c|}{ VoIP } & \multicolumn{1}{|c|}{ Video } & \multicolumn{1}{l|}{ Gaming } & \multicolumn{1}{l|}{ FTP } & Web & \\
\hline BW & \multicolumn{7}{|c|}{ Users- distribution } & Total \\
\hline 5 & 8 & 5 & 5 & 5 & 3 & 25 \\
\hline 10 & 16 & 11 & 11 & 11 & 5 & 54 \\
\hline 15 & 24 & 16 & 16 & 16 & 8 & 80 \\
\hline 20 & 33 & 22 & 22 & 22 & 11 & 109 \\
\hline BW & \multicolumn{7}{|c|}{ Application- Data Usage- Kbps } & Total \\
\hline 5 & 494 & 4306 & 268 & 1297 & 82 & 6445 \\
\hline 10 & 1066 & 9300 & 579 & 2801 & 176 & 13922 \\
\hline 15 & 1579 & 13778 & 858 & 4150 & 261 & 20625 \\
\hline 20 & 2152 & 18772 & 1168 & 5654 & 355 & 28102 \\
\hline
\end{tabular}


Table V gives the capacity of the system for DL in terms of number of users served for AD-1. The table also gives the total data usage of each application in kbps.

\section{a) Impact of Bandwidth}

Bandwidth of the system impacts the capacity of the network in that an increase in bandwidth allows a larger number of users to be served as shown in table V. It is because as the bandwidth increases the TBPS available to the RAC increases as indicated in table III. The bandwidth also has a direct impact on the number of packets for user's data. For example, in case of low bandwidth, a subframe can accommodate less amount of user's data. Consequently, more packets need to be sent to meet the data rate requirements. As a result, the protocol $\mathrm{OH}$ increases. For an instance, for $3 \mathrm{MHz}$ bandwidth and with same $64 \mathrm{QAM}-3 / 5 \mathrm{MCS}$, the protocol $\mathrm{OH}$ for video applications increases by approximately $5 \mathrm{kbps}$ compared to the protocol $\mathrm{OH}$ estimated in table IV. As a result, it reduces the capability of the network to serve the users with same bandwidth. Hence, lower bandwidth decreases the total number of users that can be supported in the network.

\section{b) Impact of Application Distribution (AD)}

Along with bandwidth employed in the cell, the application distribution also has an impact on the number of supported users. An application distribution with higher ratio of application with low bandwidth requirements results in higher number of total users in the network.

TABLE VI. NUMBER OF SUPPORTED USERS AND APPLICATIONS DATA-USAGE WITH AD-2

\begin{tabular}{|r|r|r|r|r|r|r|}
\hline & VoIP & Video & Gaming & FTP & Web & \\
\hline BW & \multicolumn{7}{|c|}{ Users- distribution } & Total \\
\hline 5 & 2 & 6 & 3 & 3 & 2 & 16 \\
\hline 10 & 3 & 13 & 7 & 7 & 3 & 33 \\
\hline 15 & 5 & 20 & 10 & 10 & 5 & 50 \\
\hline 20 & 7 & 26 & 13 & 13 & 7 & 66 \\
\hline BW & \multicolumn{7}{|c|}{ Application- Data Usage- Kbps } & Total \\
\hline 5 & 105 & 5511 & 172 & 830 & 52 & 6445 \\
\hline 10 & 217 & 11367 & 354 & 1712 & 108 & 13922 \\
\hline 15 & 329 & 17222 & 536 & 2593 & 163 & 20625 \\
\hline 20 & 441 & 23078 & 718 & 3475 & 218 & 28102 \\
\hline
\end{tabular}

Table VI indicates the capacity of the network for AD-2 given in table II. It clearly demonstrates that by increasing the distribution of streaming application from $20 \%$ to $40 \%$ the total number of users that an eNodeB can serve with a particular BW reduces. It is because the bandwidth demand of video users is very high compared to voice users. So the $A D$, which has higher percentage of application with high bandwidth demand, significantly reduces the total number of users served in a cell.

\section{c) Impact of QoS Schemes}

The above section discusses the capacity of the network for a given traffic distribution without taking any QoS scheme into consideration. Once the network is dimensioned, the demand for any specific service or mix of services can increase due to changes in population density or introduction of an event or new application. Also, the load in the core network can impose requirement on network operators to redimension the network. Whereas, by applying QoS mechanisms including $\mathrm{RAC}$ and $\mathrm{CC}$, the network operators may delay the re-dimensioning while still ensuring the QoS to connections. With the RAC, the network can effectively control the admission of each type of service to advantage both the users and the network operators. The CC determines the extent to which the load in the core network can be handled by an access network with an acceptable delay.

Let us first discuss the impact of the proposed RAC in [4]. LTE-FIAC, in times of resource scarcity, admits a connection of high priority by applying a BA algorithm. The BA algorithm stepwise degrades the bandwidth allocated to connections of low priority services. The bandwidth of a connection is allowed to be reduced only to the minimum rate stated by GBR. Hence, it enables the network to handle changes in the demand of high priority services like voice, video and online games.

Table VII shows the scenario hereafter referred as case-1, where the increase in demand is only for voice traffic. Table VII demonstrates with the use of the proposed RAC scheme, the network operator with same network configurations can gain $68 \%$ increase in terms of number of voice users supported with respect to the base scenario given in Table V. Table VII shows after the RAC admits new voice users in the network, the total number of connections of all other services are same but the overall data usage of FTP and web application reduces. This is because LTE-FIAC admits the high priority voice connections by degrading the bandwidth allocated to the low priority data flows of FTP and web to their GBR.

TABLE VII. NUMBER OF SUPPORTED USERS AND APPLICATIONS DATA-USAGE, INCREASE IN ONLY VOIP SERVICE DEMAND - (CASE-1)

\begin{tabular}{|c|c|c|c|c|c|c|}
\hline & VoIP & Video & Gaming & FTP & Web & \\
\hline BW & \multicolumn{5}{|c|}{ Users- distribution } & Total \\
\hline 5 & 24 & 5 & 5 & 5 & 3 & 41 \\
\hline 10 & 51 & 11 & 11 & 11 & 5 & 89 \\
\hline 15 & 76 & 16 & 16 & 16 & 8 & 132 \\
\hline 20 & 104 & 22 & 22 & 22 & 11 & 180 \\
\hline BW & \multicolumn{5}{|c|}{ Application- Data Usage- Kbps } & Total \\
\hline 5 & 1566 & 4306 & 268 & 267 & 39 & 6445 \\
\hline 10 & 3383 & 9300 & 579 & 576 & 84 & 13922 \\
\hline 15 & 5011 & 13778 & 858 & 854 & 125 & 20625 \\
\hline 20 & 6828 & 18772 & 1168 & 1163 & 170 & 28102 \\
\hline
\end{tabular}

Table VIII shows three cases. For all three cases, the table shows results for only the applications which undergo a change in terms of users supported. In case-2, there is an increase only in demand of video application. By applying RAC the network can gain $20 \%$ increase in terms of number of video users supported compared to the video users supported in the base scenario in Table V. In case-3, there is an increase only in demand of gaming application. The RAC 
by degrading the low priority connections can support $80 \%$ increase in terms of number of gaming users with respect to the base scenario in table $\mathrm{V}$. The increase in gaming users is much bigger than the increase in voice and video users as shown in case 1 and 2 . It is because the bandwidth requirement of online gaming application is the minimum of the three applications according to the specifications given in table II.

TABLE VIII. NUMBER OF SUPPORTED USERS AND APPLICATIONS DATA-USAGE

\begin{tabular}{|c|c|c|c|c|c|}
\hline & Case-2 & Case-3 & \multicolumn{3}{|c|}{ Case-4 } \\
\hline & Video & Gaming & $\begin{array}{l}\text { Voice } \\
0.5\end{array}$ & $\begin{array}{l}\text { Video } \\
0.25\end{array}$ & $\begin{array}{l}\text { Gaming } \\
0.25\end{array}$ \\
\hline BW & \multicolumn{5}{|c|}{ Users- distribution } \\
\hline 5 & 6 & 25 & 16 & 5 & 10 \\
\hline 10 & 13 & 54 & 34 & 11 & 22 \\
\hline 15 & 20 & 80 & 50 & 17 & 32 \\
\hline 20 & 27 & 109 & 68 & 23 & 44 \\
\hline BW & \multicolumn{5}{|c|}{ Application- Data Usage- Kbps } \\
\hline 5 & 5378 & 1341 & 1030 & 4574 & 536 \\
\hline 10 & 11617 & 2895 & 2224 & 9879 & 1158 \\
\hline 15 & 17210 & 4290 & 3295 & 14636 & 1716 \\
\hline 20 & 23448 & 5845 & 4490 & 19941 & 2338 \\
\hline
\end{tabular}

In case-4, there in is an increase in demand of all three services, voice, video and online gaming. The probability of admitting voice, video and gaming services are assumed to be $0.5,0.25$ and 0.25 , respectively. By applying RAC the network obtained $52 \%, 5 \%$ and $50 \%$ gain in terms of users supported for each application compared to users given in Table V. Table VIII also indicates the corresponding changes in total data usage of applications for each case. The data usage of FTP and web applications in case-2, 3 and 4 remains the same as given in case-1. It is because in all four cases the RAC stepwise degrades their allocated rates to their minimum rates to admit incoming high priority connections.

The results show that in times of resources scarcity, the RAC does not grant low priority FTP and web connections by degrading the connections at same priority, with a view to keep resources for incoming high priority connections [4]. So, the blocking probability (BP) of low priority services will be high compared to high priority services.

The proposed congestion control scheme in [3], defines a target operating point at an output buffer of an eNodeB and maintains the queue length around the target point. It employs a queue control function to detect congestion at the core network. In response to congestion, it applies a BA algorithm, which reduces the rate offered to the connections starting with low priority services until the queue length reaches to the define target point. It also upgrades connection's expected rate, when the queue operates below the target point. In current scenario, when an eNodeB detects the congestion, with LTE-FICC it can reduce the rate allocated to low priority FTP and web connections up to their GBR. Hence, for AD-1, LTEFICC at maximum reduce data rate requirements of a cell to around 5.4, 11.6, 17.2 and $23.4 \mathrm{Mbps}$ for 5, 10, 15 and 20
$\mathrm{MHz}$ bandwidths, respectively. An eNodeB can ensure the minimum guaranteed QoS to data flows until the capacity of core network can support its control channel rate and data channel's minimum rate offered by LTE-FICC. In situations when the capacity of the core is lower than the minim rate offered by LTE-FICC, the queue at an output buffer of an eNodeB starts building up. As a result, the QoS of connections degrades in terms of throughput and delay.

Hence, by employing these QoS schemes at an eNodeB, the network, to a certain extent, is able to manage the changes in the demand and load in the core network without the need to re-dimension. Once the RAC admits the high priority connections by degrading all low priority connections to their respective minimum rate (GBR), the $\mathrm{BP}$ of high priority services also starts increasing. The network operator can define a range of acceptable BPs for different services. Once the BP of a service such as voice or mix of service such as voice and video goes beyond the range, it can mark it as an indication to re-dimension the network. Similarly, when the capacity of a bottleneck link at the core is even lower than the minimum throughput offered by LTE-FICC, the QoS of connections degrades. The network operator can define a threshold for acceptable minimum QoS. When the QoS of the connections reduces below the minimum limit, it is set as an alert for provisioning additional resources or re-dimensioning the network. In current analysis, bandwidth allocation of only TCP based FTP and web traffic is specified by a range as shown in table II. Therefore, the BA algorithm is applied to utilize the gap between their respective MBR and GBR. The network operators depending on preferences may also vary priority and values of MBR and GBR of other applications such as video. This may enable the operators to cope with more dynamic variations in demand of high priority services such as voice and load at the core network.

\section{CONCLUSION}

The paper investigated the impacts of QoS schemes on the capacity and dimensioning of LTE networks. It presented a general and an efficient approach for network operators to determine the extent to which current network configurations with the employed QoS schemes can effectively manage the dynamic variations in the access and core side of the network. With this approach, an indication of the need for redimensioning can be presented to network operators to ensure QoS of connections in terms of throughput and delay.

\section{REFERENCES}

[1] 3GPP 36.211, "Technical Specification Group Radio Access Network; Evolved Universal Terrestrial Radio Access (E-UTRA); Physical Channels and Modulation (Release 11)."

[2] Arthur W. Berger and Yaakov Kogan, "Dimensioning Bandwidth and Connection Admission Control for Elastic Traffic in HighSpeed Communication Networks," July, 2001.

[3] Fatima Furqan and Doan B. Hoang, "LTE_FICC: A New Mechanism for Provision of QoS and Congestion Control in LTE/LTE-Advanced Networks," presented at the Mobiquitous WEWFMN 2013.

[4] Fatima Furqan, et al., "LTE/LTE-Advanced Fair Intelligent Admission Control - LTE-FIAC," IEEE International Symposium 
on a World of Wireless, Mobile and Multimedia Networks (WoWMoM 2014).

[5] Nafiz Imtiaz Bin Hamid, et al., "Coverage and Capacity Analysis of LTE Radio Network Planning considering Dhaka City," vol. 46, no. 15 , pp. 49-56, May 2012.

[6] Abdul Basit Syed, "Dimensioing of LTE Network, Description of Models and Tool, Coverage and Capacity Estimation of 3GPP Long Term Evolution radio Interface."

[7] $\mathrm{Xi} \mathrm{Li}$, et al., "Dimensioning of the LTE access network," Telecommunication Systems, vol. 52, pp. 2637-2654, 2013/04/01 2013.

[8] Fredrik Persson, "Voice over IP Realized for the 3GPP Long Term Evolution," presented at the IEEE VTC Fall' 07, Sept. 2007.

[9] Amrish Kacker, et al., "Review of efficiency and prudency of NBN Co's fibre, wireless and satellite network design," 26 Sept, 2012.

[10] Fatima Furqan and Doan B. Hoang, "Analysis of Parameters Contributing Performance and Coverage of Mobile WiMAX with Mix Traffic," in Parallel and Distributed Computing, Applications and Technologies (PDCAT), 2011 12th International Conference on, 2011, pp. 313-318.

[11] Harri Holma and Antti Toskala, LTE for UMTS: OFDMA and SCFDMA based radio access: John Wiley \& Sons, 2009.

[12] Mohammad T. Kawser, et al., "Downlink SNR to CQI Mapping for Different Multiple Antenna Techniques in LTE," International Journal of Information and Electronics Engineering, vol. 2 No.5, pp. 756-760, 2012.

[13] Telesystem Innovations Inc, "LTE in a Nutshell : System Overview," 2010.

[14] LTE Model User Guide. Available: http://idefix.dit.upm.es/download/opnet/r17.1/LTE_Model_User Guide.pdf

[15] $\quad \underline{\mathrm{http}: / / \mathrm{www} . o p n e t . c o m}$ (opnet modeler release 17.1.A).

[16] Chakchai So-In, et al., "Capacity evaluation for IEEE 802.16e mobile WiMAX," J. Comp. Sys., Netw., and Comm., vol. 2010, pp. 1-12, 2010.

[17] Petter Edstrom, "Overhead Impacts on Long-Term Evolution Radio Networks," Master of Science Thesis Stockholm, Sweden 2007.

[18] 3GPP 36.213, "Technical Specification Group Radio Access Network; Evolved Universal Terrestrial Radio Access (E-UTRA); Physical layer procedures, Release 11."

[19] Harri Holma and Antti Toskala, "LTE for UMTS : evolution to LTE-advanced," Chichester : Wiley, 2011. 